\title{
Development of active tuberculosis in patients treated with biological disease-modifying antirheumatic drugs
}

\author{
J Strong, ${ }^{1}$ MB ChB; T N Mann, ${ }^{2,3}$ BSc, PhD; G S Tarr, ${ }^{2,4,5}$ MB ChB, MMed (Int); H Reuter, ${ }^{1,2,4}$ MB ChB, PhD (D) \\ ${ }^{1}$ Division of Clinical Pharmacology, Department of Medicine, Faculty of Medicine and Health Sciences, Stellenbosch University, Cape Town, \\ South Africa \\ ${ }^{2}$ Institute of Orthopaedics and Rheumatology, Mediclinic Winelands Orthopaedic Hospital, Stellenbosch, South Africa \\ ${ }^{3}$ Division of Orthopaedic Surgery, Department of Surgical Sciences, Faculty of Medicine and Health Sciences, Stellenbosch University, Cape Town, \\ South Africa \\ ${ }^{4}$ Winelands Rheumatology Centre, Stellenbosch, South Africa \\ ${ }^{5}$ Division of Rheumatology, Department of Medicine, Faculty of Medicine and Health Sciences, Stellenbosch University, Cape Town, South Africa
}

Corresponding author: H Reuter (hr@sun.ac.za)

\begin{abstract}
Background. Biological disease-modifying antirheumatic drugs (bDMARDs) have been shown to be highly effective in the treatment of rheumatic conditions, but may increase the risk of infections. Development of tuberculosis (TB) while on bDMARD therapy is of particular concern in high TB burden settings such as Western Cape Province, South Africa.

Objectives. To describe the diagnosis, management and outcome of patients who developed active TB while receiving a bDMARD.

Results. Ten patients who screened negative for TB prior to initiation of a bDMARD subsequently developed active TB. TB was diagnosed between 10 months and 9 years from bDMARD initiation, suggesting new infection, and included 6 cases of extrapulmonary TB. All patients required multiple tests to confirm the diagnosis of TB, and all were successfully treated.

Conclusions. TB can occur in patients on bDMARD therapy despite initial screening, and may have unusual, extrapulmonary manifestations that pose a diagnostic challenge.
\end{abstract}

S Afr Med J 2022;112(2):76-80. https://doi.org/10.7196/SAMJ.2022.v112i2.16036

Biological disease-modifying antirheumatic drugs (bDMARDs) have been shown to be highly effective in the treatment of immunemediated inflammatory arthritides such as rheumatoid arthritis (RA), psoriatic arthritis (PsA) and axial spondyloarthritis (axSpA). ${ }^{[1,2]}$ However, their use has been limited by cost and the risk of opportunistic infections. ${ }^{[3-5]}$ This type of therapy is therefore reserved for patients who have failed treatment with conventional synthetic disease-modifying antirheumatic drugs (sDMARDs). ${ }^{[1,2]}$

bDMARDs are classified according to their molecular target. ${ }^{[1]}$ Although the mechanism of action will vary accordingly, a common way of grouping bDMARDs has been to divide them into tumour necrosis factor alpha (TNF- $\alpha$ ) inhibitors and non-TNF- $\alpha$ inhibitors. ${ }^{[1,2]}$ TNF- $\alpha$ is a key inflammatory cytokine in the immunopathogenesis of RA and various other rheumatic conditions. However, it also plays an important role in the containment and elimination of Mycobacterium tuberculosis. Tuberculosis (TB) is therefore of particular concern among patients receiving TNF- $\alpha$ inhibitors, a premise supported by several reports of increased risk of active TB among patients receiving TNF- $\alpha$ inhibitors $v$. those receiving non-TNF- $\alpha$-inhibiting bDMARDs. ${ }^{[6-9]}$ Furthermore, within the class of TNF- $\alpha$ inhibitors, the monoclonal antibodies infliximab, adalimumab and golimumab have been found to carry a slightly higher risk of TB than the dimeric receptor fusion protein, etanercept. ${ }^{[10,11]}$ Initially, TNF- $\alpha$ inhibitors were the only type of bDMARD available for treatment of PsA and axSpA, whereas bDMARDs for the treatment of RA included both TNF- $\alpha$-inhibitor and non-TNF- $\alpha$-inhibitor options. ${ }^{[1,2]}$

South Africa (SA) has one of the highest burdens of TB worldwide, ${ }^{[12]}$ so the risk of TB in patients receiving a bDMARD is of particular concern in our setting. A study based on data from the South African Biologics Registry (SABIO) reported that 96 patients developed TB between 1999 and 2017 and inferred a TB incidence of 1240 cases per 100000 person-years among patients receiving bDMARDs for rheumatic diseases. ${ }^{[4]}$ Notably, the incidence of TB among users of TNF- $\alpha$ inhibitors in the SABIO registry was 1387 per 100000 person-years - at least eight times higher than that of the British (British Society for Rheumatology Biologics Register, BSRBR), French (French Research Axed on Tolerance of Biotherapies registry, RATIO) and Spanish (Base de Datos de Productos Biólogicos de la Sociedad Española de Reumatologia, BIOBADASER) registries, where the TB incidence ranged from 106 to 172 per 100000 personyears among users of TNF- $\alpha$ inhibitors. ${ }^{[4,11]}$

Although the SABIO study showed a TB incidence markedly higher than those of European registries, the data were largely reflective of patients treated in the private healthcare sector. ${ }^{[4]}$ This group would typically have had higher socioeconomic status and lower TB risk than the wider SA population, inferring that TB incidence among bDMARD users in the public sector may be even higher. This premise was supported by a study that investigated the incidence of TB among 609 patients who received bDMARD therapy in the Western Cape Province public health sector between 2007 and 2018. ${ }^{[5]}$ The study included use of bDMARDs for both immune-mediated inflammatory disease and haematological malignancy, precluding direct comparison with the registry data. Nevertheless, the findings suggested a substantially increased risk of TB among patients receiving bDMARDs in the public sector, including an incidence of 2819 cases per 100000 person-years among users of TNF- $\alpha$ inhibitors. ${ }^{[5]}$ 
The aforementioned studies demonstrated that the risk of developing TB during treatment with a bDMARD may be substantially higher in SA than in developed countries with a low TB burden. ${ }^{[4,5]}$ However, to our knowledge there has been little further investigation of this relevant topic in our setting. This case series aimed to add to the limited existing knowledge by describing the diagnosis, management and outcome of patients who developed TB while receiving bDMARD therapy at a private rheumatology centre in the Western Cape.

\section{Case series}

The case series involved 10 patients with rheumatic conditions who developed active TB while receiving treatment with a bDMARD. All patients were seen at a private rheumatology centre in the Cape Winelands district of the Western Cape. The rheumatology centre has a patient base of $\sim 3000$ individuals, of whom $\sim 300$ are on bDMARD treatment. The cases of TB presented in the current series were prospectively identified by the treating rheumatologists between 2006 and 2020. TB episodes were reported in SABIO and were also recorded in a practice database. Seven patients provided signed consent for the use of their medical information in the case series, and motivation for waiver of consent was accepted for the remaining
3 cases. The study was approved by a university health research ethics committee (ref. no. C21/07/020).

\section{Patient demographic and clinical characteristics}

Patient demographics, clinical characteristics and TB screening results are shown in Table 1. All patients had undergone mandatory TB screening prior to bDMARD initiation, of whom 8 had no screening results suggestive of TB: 3 patients had a negative tuberculin skin test (TST), a negative interferon gamma release assay (IGRA) test and a normal chest radiograph; 4 had a negative TST and a normal chest radiograph; and 1 had a negative TST and radiographic findings indicative of known interstitial lung disease but not of TB. Of the 2 remaining patients, 1 had a positive TST and radiographic findings suggestive of $\mathrm{TB}$; however, these observations were consistent with a previous TB episode that had been successfully treated. The other patient had a positive TST and a normal chest radiograph and was prescribed isoniazid preventive therapy (IPT) prior to commencing a bDMARD. This patient was the only one of the 10 patients who received IPT.

DMARD and steroid use among the patients are shown in Table 2. At the time of developing TB, 7 patients were receiving adalimumab, a TNF- $\alpha$ inhibitor. Two of these patients had previously been treated

Table 1. Patient demographics, clinical characteristics, pretreatment TB screening and isoniazid prophylaxis

\begin{tabular}{|c|c|c|c|c|c|c|c|c|}
\hline \multirow[b]{2}{*}{ Patient no. } & \multirow{2}{*}{$\begin{array}{l}\text { Age at TB } \\
\text { diagnosis (years) }\end{array}$} & \multirow[b]{2}{*}{ Gender } & \multirow{2}{*}{$\begin{array}{l}\text { Rheumatic } \\
\text { disease }\end{array}$} & \multirow[b]{2}{*}{ Comorbidities } & \multicolumn{3}{|c|}{ Pre-bDMARD TB screening } & \multirow{2}{*}{$\begin{array}{l}\text { Isoniazid } \\
\text { prophylaxis }\end{array}$} \\
\hline & & & & & TST & IGRA & CXR & \\
\hline 1 & 46 & $\mathrm{M}$ & axSpA & Hypertension & Negative & Negative & Normal & No \\
\hline 2 & 61 & $\mathrm{~F}$ & RA & None & Negative & Negative & Normal & No \\
\hline 3 & 42 & $\mathrm{~F}$ & RA & None & Negative & Negative & Normal & No \\
\hline 4 & 82 & $\mathrm{~F}$ & RA & $\begin{array}{l}\text { Lung fibrosis } \\
\text { Previous stroke }\end{array}$ & Negative & $\mathrm{n} / \mathrm{d}$ & ILD & No \\
\hline 5 & 22 & $\mathrm{~F}$ & PsA & Obesity & Negative & $\mathrm{n} / \mathrm{d}$ & Normal & No \\
\hline 6 & 40 & $\mathrm{~F}$ & RA & None & Negative & $\mathrm{n} / \mathrm{d}$ & Normal & No \\
\hline 7 & 36 & M & axSpA & None & Negative & $\mathrm{n} / \mathrm{d}$ & Normal & No \\
\hline 8 & 52 & $\mathrm{~F}$ & RA & $\begin{array}{l}\text { Asthma } \\
\text { Previous PTB }\end{array}$ & Positive & $\mathrm{n} / \mathrm{d}$ & Suggestive of TB & $\mathrm{No}^{*}$ \\
\hline 9 & 50 & M & RA & $\begin{array}{l}\text { Hypertension } \\
\text { High cholesterol }\end{array}$ & Positive & $\mathrm{n} / \mathrm{d}$ & Normal & Yes \\
\hline 10 & 29 & $\mathrm{~F}$ & RA & None & Negative & $\mathrm{n} / \mathrm{d}$ & Normal & No \\
\hline
\end{tabular}

$\mathrm{TB}=$ tuberculosis; $\mathrm{M}$ = male; $\mathrm{F}=$ female; axSpA = axial spondyloarthritis; $\mathrm{RA}=$ rheumatoid arthritis; $\mathrm{Ps} \mathrm{A}=$ psoriatic arthritis; $\mathrm{PTB}=$ pulmonary tuberculosis; $\mathrm{bDMARD}=$ biological diseasemodifying antirheumatic drug; TST = tuberculin skin test; IGRA = interferon gamma release assay; $\mathrm{n} / \mathrm{d}=$ not done; ILD = interstitial lung disease. ${ }^{*} \mathrm{~TB}$ screening results attributed to previous TB episode.

Table 2. Disease-modifying antirheumatic drug and steroid use at the time of TB diagnosis

\begin{tabular}{|c|c|c|c|c|}
\hline \multirow[b]{2}{*}{ Patient no. } & \multicolumn{2}{|c|}{ bDMARDs } & \multirow[b]{2}{*}{ sDMARDs at TB diagnosis } & \multirow[b]{2}{*}{ Steroid use at TB diagnosis } \\
\hline & Prior & At TB diagnosis & & \\
\hline 1 & - & Adalimumab & None & None \\
\hline 2 & Etanercept & Adalimumab & Methotrexate & None \\
\hline 3 & - & Adalimumab & Methotrexate & Prednisone \\
\hline 4 & Etanercept & Adalimumab & Methotrexate & None \\
\hline 5 & - & Adalimumab & Leflunomide & None \\
\hline 6 & - & Adalimumab & Methotrexate & None \\
\hline \multirow[t]{2}{*}{7} & Etanercept & Adalimumab & None & None \\
\hline & Infliximab & & & \\
\hline 8 & - & Rituximab & None & Inhaled corticosteroids \\
\hline 9 & - & Etanercept & Methotrexate & None \\
\hline 10 & Adalimumab & Tocilizumab & None & None \\
\hline
\end{tabular}


with etanercept and 1 had previously been treated with etanercept and infliximab, which are also TNF- $\alpha$ inhibitors. The remaining 3 patients were receiving rituximab, etanercept and tocilizumab, respectively, at the time of developing TB. Rituximab is a B-celldepleting bDMARD and tocilizumab is a monoclonal antibody interleukin-6 receptor inhibitor, so these bDMARDs represent the only two non-TNF- $a$ inhibitors used by affected patients at the time of TB diagnosis. Six patients were receiving an sDMARD in addition to bDMARD therapy, and 2 patients were using steroids at the time of TB diagnosis (Table 2).

\section{Diagnosis}

Details of TB diagnosis are shown in Table 3. The time for which the patient had been receiving bDMARD therapy prior to developing active TB varied considerably, ranging from 10 months for patient 8 to 9 years 6 months for patient 7 . However, 8 of the 10 patients had been on a bDMARD for at least 2 years 11 months at the time of diagnosis.

A notable feature of the case series was that 6 of the 10 patients had extrapulmonary manifestations of TB, including unusual sites of infection. Four patients had exclusively extrapulmonary TB: patient 1 developed TB of the peritoneum and mesenteric lymph nodes, which was diagnosed following a peritoneal biopsy and peritoneal fluid aspirate; patient 2 developed TB of the larynx and cervical lymph nodes, which was diagnosed following a vocal cord and cervical lymph node biopsy; patient 6 developed TB of the pleura, which was diagnosed following a chest radiograph, pleural biopsy and pleural fluid aspirate; and patient 7 developed TB of the mediastinal lymph nodes and possibly of the meninges, which was diagnosed following a lumbar puncture, chest computed tomography scan and mediastinal lymph node biopsy. Pulmonary TB was excluded by chest imaging in these 4 patients.

The other 2 patients with extrapulmonary TB had evidence of concurrent pulmonary involvement: patient 3 developed TB of the extensor tendon of the wrist and of the lungs, which was diagnosed following a biopsy of the synovium of the joint and a positive sputum culture; and patient 9 developed disseminated TB involving the lungs, axillary lymph nodes and pericardium, which was diagnosed following chest imaging, an axillary lymph node biopsy and a pleural tap. The remaining 4 patients had pulmonary TB only, in each case diagnosed following suggestive chest imaging and smear- or culturepositive sputum.

\section{Management and outcome}

Upon TB diagnosis, the bDMARD was stopped and TB treatment initiated. All patients were treated according to the TB drug regimen described in the national TB management guidelines, ${ }^{[13]}$ and most received sDMARD treatment throughout the course of TB treatment (Table 3). Seven patients completed the standard 6-month course of TB treatment, whereas treatment was extended in patients 4, 7 and 9 under the following circumstances. Patient 4 had pulmonary TB with radiographic evidence of cavitation and a history of previously treated TB. She had a delayed response to TB treatment and her treatment was therefore extended to 10 months. Patient 7 had mediastinal lymph node TB and possible TB meningitis. Upon suspension of adalimumab, he developed severe immune reconstitution inflammatory syndrome and required corticosteroid therapy in addition to the anti-TB drugs. After 6 months of TB treatment, his mediastinal lymph nodes were still enlarged and his $\mathrm{TB}$ treatment was extended to 12 months. Patient 9 had disseminated TB and owing to a slow response his TB treatment was prolonged to 9 months of treatment, which resulted in complete cure. All 10 patients were successfully cured of TB with only one known complication, development of a post-TB aspergilloma in a lung cavity in patient 4 .

Five patients resumed bDMARD therapy after completing TB treatment, including 2 on rituximab, 1 on abatacept, 1 on adalimumab and 1 on tocilizumab (Table 3). Patient 6 was trialled on abatacept but was subsequently switched to tocilizumab and then to rituximab, following poor efficacy and an infusion reaction, respectively. Patient 7 resumed treatment with adalimumab 3 months after completion of TB treatment and remained on adalimumab and INH preventive therapy until March 2020. At the time of his TB episode, TNF- $\alpha$ inhibitors were the only available bDMARDs for spondyloarthritis. When the interleukin-17A (IL-17A) inhibitor secukinumab was licensed in SA, his treatment was changed from adalimumab to secukinumab, which is thought to be associated with a lower risk of TB. The remaining 5 patients did not resume bDMARD therapy after the TB episode, but were successfully managed with various sDMARDs (Table 3). At most recent follow-up, all patients had achieved disease remission for their respective rheumatic conditions and had experienced no further episodes of TB.

\section{Discussion}

The first feature of the current case series was the time period between initiating a bDMARD and development of active TB, which ranged from 10 months to $>9$ years among the patients. TB occurring within the first 6 months of initiating a bDMARD is normally regarded as reactivation $\mathrm{TB}$, whereas the longer time frames of the current series are suggestive of new-onset TB. The likelihood of new-onset TB is also supported by the fact that patients screened negative for latent TB prior to initiating a bDMARD or were prescribed IPT as indicated. TB screening carries the risk of false-negative results, especially in the case of TST tests among patients with rheumatic conditions, who would have been receiving immune-suppressing sDMARDs. ${ }^{[14]}$ However, the screening appears to have been adequate for the current patients, and the minimum TB screening requirements of the South African Rheumatism and Arthritis Association (SARAA) guidelines ${ }^{[14]}$ - a TST and a chest radiograph - were adhered to in all cases.

The approach to IPT for patients initiating bDMARD therapy may differ between the private and public sectors in SA. Only one of the current private sector patients was initiated on IPT, following a positive TST, and the IPT had a fixed duration of 9 months. However, in some public hospitals in SA, patients may receive IPT throughout the duration of treatment with a bDMARD. This approach is presumably seen as a necessary precaution in view of the relatively high underlying TB risk in the public sector population. ${ }^{[5]}$ The SARAA guidelines note that 9 months of IPT may be appropriate for patients at very high risk of TB who are due to commence antiTNF- $\alpha$ therapy, irrespective of TST or IGRA test results. ${ }^{[14]}$ However, at present there is little evidence with which to evaluate the risks and benefits of long-term IPT while receiving a TNF- $\alpha$ inhibitor or a nonTNF- $\alpha$ inhibitor. This constitutes an important area for further study.

A further feature of the current case series was that 6 of 10 patients had extrapulmonary manifestations of TB, including a number of unusual sites of TB disease such as the extensor tendon, the larynx and the meninges. Reports suggest that typically only $15 \%$ of all $\mathrm{TB}$ cases in SA are classified as extrapulmonary, ${ }^{[12]}$ so the proportion in the current series is abnormally high. However, this observation is in keeping with previous reports of increased extrapulmonary TB among bDMARD users, including $38 \%$ of cases with extrapulmonary TB in the SABIO study. ${ }^{[4]}$ 


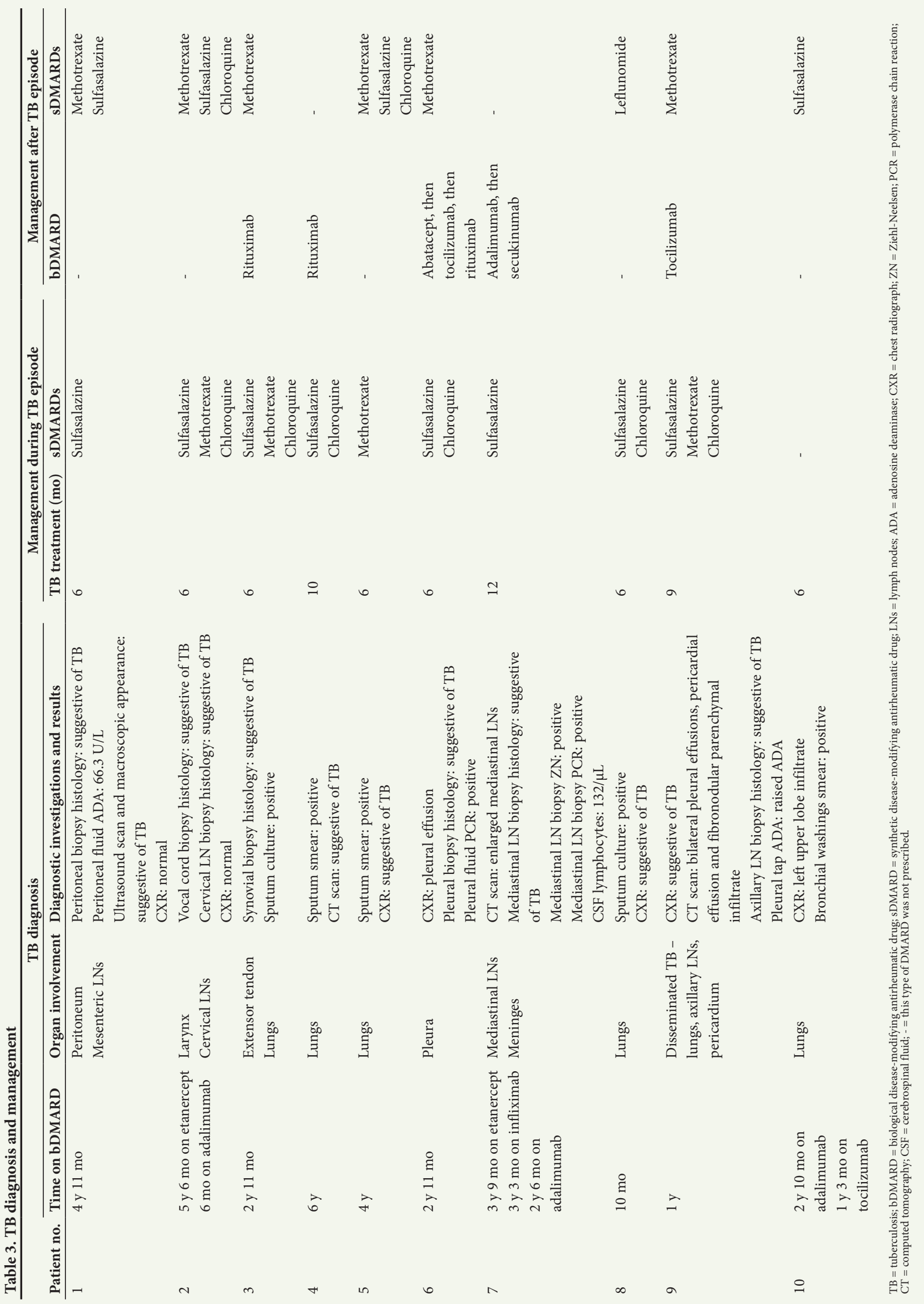


Increased extrapulmonary $\mathrm{TB}$ may suggest poor containment of $\mathrm{TB}$ in the lungs, and would be in keeping with reduced TNF- $\alpha$ function to contain TB in the lung via granuloma formation. Of the 8 patients who were receiving a TNF- $\alpha$ inhibitor at the time of TB diagnosis, 6 had extrapulmonary involvement and 2 had exclusively pulmonary TB. Conversely, the 2 patients receiving non-TNF- $\alpha$ inhibitors both developed exclusively pulmonary $\mathrm{TB}$. We are not aware of studies that have directly assessed the relative risk of extrapulmonary TB when receiving TNF- $\alpha$ inhibitors v. non-TNF- $\alpha$ inhibitors. However, the current observations were consistent with previous reports of a large proportion of extrapulmonary $\mathrm{TB}$ among individuals who developed TB while receiving a TNF- $\alpha$ inhibitor. ${ }^{[11,15,16]}$ For example, a study from the BSRBR reported that 25 of 40 (62\%) of TB cases in the anti-TNF- $\alpha$ cohort were extrapulmonary. ${ }^{[11]}$ Extrapulmonary TB can present a diagnostic challenge, and cases in the current series required a number of investigations before TB was diagnosed. Nevertheless, all cases in the series, both pulmonary and extrapulmonary, were ultimately histologically and/ or bacteriologically confirmed and successfully treated with a minimal number of complications.

Little is known about the safety of resuming bDMARD therapy after a TB episode. However, 5 patients in the current case series did resume treatment with a bDMARD, albeit with precautions. In most cases, this involved selection of a non-TNF- $\alpha$-inhibitor bDMARD, which is associated with lower risk of TB. ${ }^{[4]}$ More specifically, 4 patients who had been on a TNF- $\alpha$ inhibitor prior to the TB diagnosis were switched to a non-TNF- $\alpha$ inhibitor. Only one patient resumed bDMARD therapy with a TNF- $\alpha$ inhibitor after the TB diagnosis. However, this patient remained on concurrent IPT to reduce the risk of TB before later switching to secukinumab, an IL-17a inhibitor. Notably, non-TNF- $\alpha$-inhibitor bDMARDs were licensed for use in RA at an earlier stage than for other rheumatic conditions such as axSpA and PsA and influenced the DMARD treatment options for each patient over the course of the study period. Nevertheless, both those resuming bDMARDs and those reverting to sDMARDs achieved disease remission and have had no further TB episodes to date.

A strength of the current case series was that we were able to provide detailed information on screening and diagnostic test results along with pre- and post-TB DMARD use among the patients involved. A limitation was that we were not able to obtain reliable information on steroid use prior to initiation of bDMARD therapy. Two of the patients were known to be using steroids at the time of TB diagnosis, a factor that may help to explain TB susceptibility in these particular individuals. However, the possible significance of prior steroid exposure for TB risk in this patient population constitutes an important aspect for inclusion in future studies.

\section{Conclusions}

The current case series adds to limited existing knowledge on the development of active TB while receiving bDMARD therapy in a high TB burden setting. It highlights that ostensibly new-onset TB may occur several years after bDMARD initiation and have unusual extrapulmonary manifestations that pose a diagnostic challenge. Clinicians managing patients on bDMARD therapy are therefore encouraged to maintain a high index of suspicion for TB, even if initial screening tests were negative. Finally, the cases presented offer encouraging evidence that $\mathrm{TB}$ can be successfully treated and certain bDMARD therapies resumed.

\section{Teaching points}

- Active TB developed up to several years after bDMARD initiation.

- Some patients had extrapulmonary TB with uncommon disease sites.

- Multiple diagnostic tests were required.

- All patients were successfully treated.

- Several patients resumed bDMARD therapy after completion of TB therapy.

Declaration. None.

Acknowledgements. We thank Sister Liesl Segal and Ms Carmen Flagg for their kind assistance during the study.

Author contributions. JS: collected the data and wrote the first draft of the manuscript. TNM: revised and further developed the manuscript. GST: assisted with data collection and reviewed the manuscript. HR: conceptualised the case series, assisted with data collection and reviewed the manuscript. All authors approved the final version of the manuscript. Funding. None.

Conflicts of interest. None.

1. Tarr G, Hodkinson B, Reuter H. Superheroes in autoimmune warfare: Biologic therapies in current SA practice. S Afr Med J 2014;104(11):787-791. https://doi.org/10.7196/SAMJ.8947

2. Harmse L, Reuter H. An overview of the biological disease modifying drugs available for arthritic conditions in South Africa. S Afr Fam Pract 2016;58(6):6-10. https://doi.org/10.4102/safp.v58i6.4595 3. Ai JW, Zhang S, Ruan QL, et al. The risk of tuberculosis in patients with rheumatoid arthritis treated with tumor necrosis factor- $\alpha$ antagonist: A metaanalysis of both randomized controlled trials an registry/cohort studies. J Rheum 2015;42(12):2229-2237. https://doi.org/10.3899/jrheum.150057

4. Pettipher C, Benitha R. Tuberculosis in biologic users for rheumatic diseases: Results from the South African Biologics Registry (SABIO). Ann Rheum Dis 2020;79(2):292-299. https://doi.org/10.1136/ annrheumdis-2019-216128

5. Du Toit T, Esterhuizen TM, Tiffin N, Abulfathi AA, Reuter H, Decloedt EH. Incident tuberculosis disease in patients receiving biologic therapies in the Western Cape, South Africa from 2007 to 2018. BMC Infect Dis 2020;20(1):900. https://doi.org/10.1186/s12879-020-05624-0

6. Cantini F, Nannini C, Niccoli L, Petrone L, Ippolito G, Goletti D. Risk of tuberculosis reactivation in Cantini F, Nannini C, Niccoli L, Petrone L, Ippolito G, Goletti D. Risk of tuberculosis reactivation in patients with rheumatoid arthritis, ankylosing spondylitis, and psoriatic arthritis receiving non-anti-
TNF-targeted biologics. Mediators Inflamm 2017;2017:8909834. https://doi.org/10.1155/2017/8909834 TNF-targeted biologics. Mediators Inflamm $2017 ; 2017: 8909834$. https:///doi.org/ $10.1155 / 2017 / 8909834$
7. Soare A, Gheorghiu AM, Aramă V, et al. Risk of active tuberculosis in patients with inflammatory . Soare A, Gheorghiu AM, Aramă V, et al. Risk of active tuberculosis in patients with inflammatory
arthritis receiving TNF inhibitors: A look beyond the baseline tuberculosis screening protocol. Clin Rheumatol 2018;37:2391-2397. https://doi.org/10.1007/s10067-017-3916-y

8. Zhang Z, Fan W, Yang G, et al. Risk of tuberculosis in patients treated with TNF- $\alpha$ antagonists: A systematic review and meta-analysis of randomised controlled trials. BMJ Open 2017;7(3):e012567. https://doi.org/10.1136/bmjopen-2016-012567

9. Ai JW, Zhang S, Ruan QL, et al. The risk of tuberculosis in patients with rheumatoid arthritis treated with tumor necrosis factor- $\alpha$ antagonist: A metaanalysis of both randomized controlled trials and registry/cohort studies. J Rheum 2015;42(12):2229-2237. https://doi.org/10.3899/jrheum.150057

10. Tubach F, Salmon D, Ravaud P, et al. Risk of tuberculosis is higher with anti-tumor necrosis factor monoclonal antibody therapy than with soluble tumor necrosis factor receptor therapy: The threeyear prospective French Research Axed on Toleran

2009;60(7):1884-1894. https://doi.org/10.1002/art.24632 Dixon WG, Hyrich KL, Watson KD, et al. Drug-specific risk of tuberculosis in patients with rheumatoid
arthritis treated with anti-TNF therapy: Results from the British Society for Rheumatology Biologics arthritis treated with anti-TNF therapy: Results from the British Society for Rheumatology Biolog
Register (BSRBR). Ann Rheum Dis 2010;69:522-528. https://doi.org/10.1136/ard.2009.118935

Register (BSRBR). Ann Rheum Dis 2010;69:522-528. https://doi.org/10.1136/ard.2009.118935
12. World Health Organization. Global tuberculosis report 2019. Geneva: WHO, 2019. https://www.who. int/teams/global-tuberculosis-programme/tb-reports/global-report-2019 (accessed 28 July 2021).

13. National Department of Health, South Africa. National Tuberculosis Management Guidelines 2014 Pretoria: NDoH, 2014 http://www.kznhealth.gov.za/family/NTCP_Adult_TB_Guidelines_2014.pdf (accessed 28 July 2021)

14. Hodkinson B, van Duuren E, Pettipher C, Kalla AA. South African recommendations for the management of rheumatoid arthritis: An algorithm for the standard of care in 2013. S Afr Med 2013;103(8):577-585. https://doi.org/10.7196/SAMJ.7047

15. Kim HW, Park JK, Yang JA, et al. Comparison of tuberculosis incidence in ankylosing spondylitis and rheumatoid arthritis during tumor necrosis factor inhibitor treatment in an intermediate burden area. Clin Rheumatol 2014;33(9):1307-1312. https://doi.org/10.1007/s10067-013-2387-z

16. Bum Chung K, Young Lee E, Pil Im J, Koo Han S, Yim J-J. Clinical characteristics and treatment responses of patients who developed tuberculosis following use of a tumor necrosis factor- $\alpha$ inhibitor. Korean J Intern Med 2013;28(2):174-179. https://doi.org/10.3904/kjim.2013.28.2.174 